\title{
The frequency principle for comparative judgments'
}

\author{
ALLEN PARDUCCI AND RUTH HAUGEN \\ UNIVERSITY OF CALIFORNIA, LOS ANGELES
}

This experiment supplements a recent study of the effects of an interpolated weight upon memory for a standard (Parducci, Marshall, \& Degner, 1966). The earlier data suggested that the stimulus-averaging approach as represented by the theory of adaptation level should be modified to incorporate the principle that $S$ s use altermative categories with equal frequency. The present data support this modification by demonstrating that even when the stimulus mean is held constant, judgments are affected by the relative frequencies of the comparison stimuli.

The method of constant stimulus differences provides a deceptively simple procedure for the study of memory. If the traditional point of subjective equality, PSE, is also the remembered value of the standard, the method provides a direct index of the effects of different conditions upon memory. For example, an unjudged stimulus can be interpolated between the standard and comparison stimulus. The effects of the interpolation upon the memory for the standard can be deduced from the theory of adaptation level (Helson, 1964). Since the theory makes PSE a weighted average of the stimuli affecting judgment, the predicted effect of the interpolated stimulus is to pull PSE and thus the memory for the standard toward its own value.

A recent experiment with lifted weights (Parducci, Marshall, \& Degner, 1966) demonstrated that although PSE shifts toward the value of an immediately preceding interpolation, it shifts away from the values of stimuli interpolated on other trials in the same experimental session. This contradicts the theory of adaptation level.

An alternative model was developed to handle these discrepant results without discarding the stimulusaveraging approach to memory. Thus, this model defines $M$, the memory for the standard, as an empirically weighted mean of the stimuli affecting judgment. However, it assumes an equal-frequency principle that separates PSE from $M$. In this frequency model, PSE is a weighted average of $M$ and the value, $F$, that PSE would have if the categories were used with equal frequency: $P S E=a M+(1-a) F$, with a an empirical constant between 0 and 1 .

Although PSEs from 11 different conditions were consistent with the frequency model, the equal-frequency assumption could not be checked directly. This was because the comparison stimuli were the same for all conditions in the previous experiment, and only the stimuli interpolated between the standard and comparison stimuli were varied. The present report describes the effects of independent variation of the comparison stimuli. With the mean of the stimuli and thus $M$ kept constant, the frequency model predicts that PSE will vary directly with $F$.

\section{Method}

Details of the procedure are presented in the original report which the present study replicates except with respect to the weights and frequencies of the comparison stimuli. Each trial began with a 109-gm standard, followed by either a 77- or 93-gm interpolation, and ended with one of eight comparison weights. The task was simply to judge whether the third weight was "heavier" or "lighter" than the first.

For the low-F condition, the comparison weights were $84,93,101,109,117,141,149$, and $157 \mathrm{gm}$. These were presented 15, 16, 3, 3, 1, 2, 1, and 7 times, respectively, in each of the seven successive blocks of 48 trials. For the high-F condition, the comparison weights were $52,60,68,93,101,109$, 117 , and $125 \mathrm{gm}$, with respective frequencies of 7,1 , $2,1,3,3,16,15$. These mirror-image, J-shaped distributions have similar geometric means, 101.9 and 100.5. The geometric mean of the rectangular distribution of comparison stimuli used in the previous experiment was 103.9. The arithmetic means were approximately 105 for all three distributions. However, the new values of $F$ were 97.5 and 112.5, as compared with the 105 of the earlier experiment.

The values of $F$ were determined by the method of successive approximation described by Parducci (1965). A hypothetical psychometric function was drawn on probit paper as a straight line with the slope set so that $\mathrm{SD}=14 \mathrm{gm}$. This was close to the empirical SD for the conditions in which the same interpolated weights had been used in the previous experiment. The line was then shifted to the left or right until the cumulated products of the proportions of "heavier" judgments and the comparison frequencies equaled one-half the total number of trials.

Each of the two comparison series was presented to a separate group of 10 undergraduates. Both the $77-$ and $93-\mathrm{gm}$ interpolations were presented to every $S$, each for half the trials. The sequence of interpolated and comparison stimuli was randomized within each block.

\section{Results and Discussion}

Figure 1 presents the most important feature of the results. The overall proportions of "heavier" 


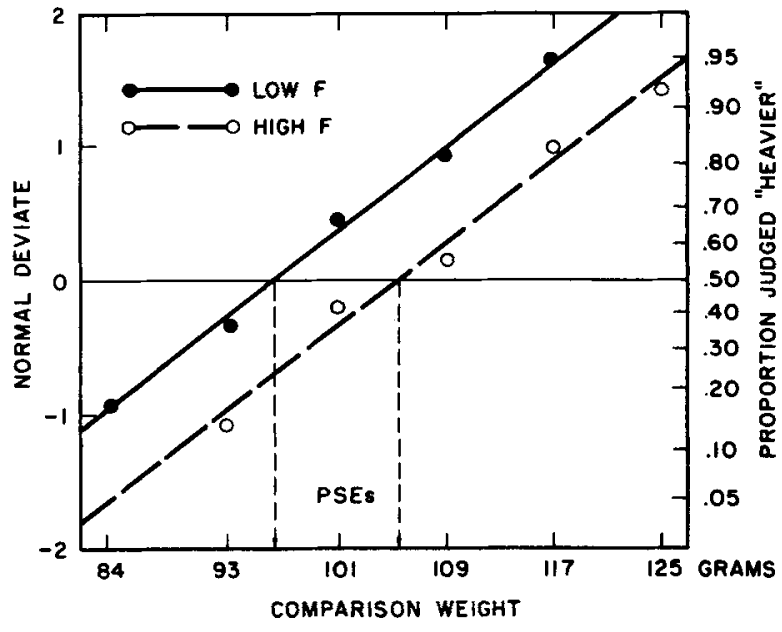

Fig. 1. Normal deviates corresponding to the proportions of "heavier" judgments of the comparison weights, tabulated across the two conditions of interpolation.

judgments have been tabulated across the two conditions of interpolation, with each PSE determined by the zero point from the least-squares fit to the corresponding normal deviates. PSE varies directly with F. The difference in PSE is almost $9 \mathrm{gm}, 1 . e ., .6$ of the difference in F. This suggests that in terms of the frequency model, the tendency toward equal frequencies is a greater influence upon judgment than is $M$, the mean of the stimuli affecting judgment.

In the previous study, separate PSEs were computed for each interpolated stimulus for each $S$. However, the wide range of the values and also of the frequencies of the comparison stimuli in the present experiment produce distressingly irregular psychometric functions. Some of the comparison weights were always discriminated so that they could not be used in determining PSE, and the infrequency with which some were presented greatly lowered the reliability of judgment. For example, a least-squares fit to one of these individual functions yielded a negative PSE. The medians of the $10 \mathrm{PSEs}$ were 95.3 and 103.0 for the low-F conditions following the 77- and 93-gm interpolations, respectively. For the high-F conditions, the median PSEs were 103.2 and 108.3. As predicted by the frequency model, these straddle the corresponding PSEs from the previous study, 99.6 and 104.6.

Another measure was also used to evaluate the effects of the experimental conditions. The 101- and 109-gm comparison weights had each been presented 21 times in each of the four conditions and were both close to the group PSEs. The proportions of times that they were judged "heavier" were used as the raw scores for an analysis of variance in which the value of the interpolated weight and the value of $F$ were independent variables. As in the previous study, these scores were significantly higher following the lighter interpolated weight $(p<.005)$, and this effect did not interact significantly with the effect of $F$. The latter was also statistically significant $(p<.025)$.

In supporting the frequency model for comparative judgments, the present data also suggest a wider application for the range-frequency theory on which it is based (Parducci, 1965). This theory was developed to describe contextual effects with absolute category judgments. Its support has come from experiments in which judgments like "heavy" and "light" are affected by the relative frequencies with which different stimuli are presented without an explicit standard. Since a standard was specified and repeatedly lifted in the present task, one might not have expected Ss to balance their judgment frequencies. However, the present data are consistent with the previous study in suggesting that the frequency principle also applies to comparative judgments.

\section{References}

Helson, H. Adaptation-level theory. New York: Harper \& Row. 1964. Parducci, A. Category judgment: A range-frequency model. Psychol. Rev., 1965, 72, 407-418.

Parducci, A., Marshall, L. M., \& Degner, M. Interference with memory for lifted weight. Percept. \& Psychophys., 1966, 1, 8386.

\section{Mote}

1. This research supported by Public Health Service Grant HD00923 and National Science Foundation Grant GB-1768.

(Accepted for publication December 7, 1966.) 